Gas Dynamics 
Abraham Achterberg

\section{Gas Dynamics}

An Introduction with Examples from Astrophysics and Geophysics 


\author{
Abraham Achterberg \\ Astronomy Department, Institute \\ for Mathematics, Astrophysics \\ and Particle Physics \\ Radboud University Nijmegen \\ Nijmegen, Gelderland \\ The Netherlands
}

ISBN 978-94-6239-194-9

ISBN 978-94-6239-195-6 (eBook)

DOI 10.2991/978-94-6239-195-6

Library of Congress Control Number: 2016940342

(C) Atlantis Press and the author(s) 2016

This book, or any parts thereof, may not be reproduced for commercial purposes in any form or by any means, electronic or mechanical, including photocopying, recording or any information storage and retrieval system known or to be invented, without prior permission from the Publisher.

Printed on acid-free paper 


\section{Foreword}

The main objective of this book is to lay the foundations of gas and fluid dynamics. It does so by developing the basic equations from scratch, building on the (assumed) knowledge of students of Classical Mechanics. In this way, we can consider the mathematical properties of flows and discuss such things as conservation laws, perturbation analysis and waves, shocks, etc. Often we will consider ideal fluids and gases, where the influence of internal friction (called viscosity) can be neglected. Viscous flows will be discussed when we consider flows around obstacles and shocks.

Many of the examples used to illustrate various processes come from astrophysics, but now and then, I will look at geophysical phenomena.

This subject is a challenging one: In order to fully understand the intricacies of fluid mechanics/gas dynamics, one needs to know about scalars, vectors and simple (rank 2) tensors, and the vector/tensor analysis that comes with it. I can only hope that this book may serve as a readable introduction to a rich subject.

Nijmegen

Abraham Achterberg

December 2015 


\section{Contents}

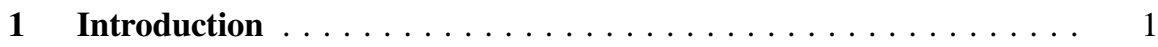

$1.1 \quad$ Scope of this Book. .................. 2

2 From Newton to Euler and Navier-Stokes. . . . . . . . . . . . 5

$2.1 \quad$ The Continuum Description. ................ 5

2.2 Eulerian and Lagrangian Time Derivatives . . . . . . . 8

2.2.1 Eulerian and Lagrangian Change . . . . . . . . . 10

$2.3 \quad$ Pressure of an Isotropic Gas . . . . . . . . . . . . . 10

2.3.1 The Stress Tensor due to Thermal Motion...... 12

2.3.2 The Case of an Isotropic Fluid or Gas in Equilibrium ..................... 14

2.4 The Euler and the Navier-Stokes Equations . . . . . . . . . 18

2.5 Pressure, Temperature and the Internal Energy. . . . . . . . 19

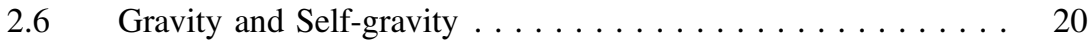

$2.7 \quad$ Mass Conservation and the Continuity Equation. . . . . . . 21

2.7.1 Equation of Motion for a Material Curve....... . 21

2.7.2 Material Volumes................. 22

2.7.3 Mass Conservation and the Continuity Equation... . 25

2.8 The Adiabatic Gas Law . . . . . . . . . . . . . . . . 27

2.8.1 The Polytropic Gas Law, the Specific Heat Coefficients and the Isothermal Gas .......... 28

2.9 Application: The Isothermal Sphere and Globular Clusters. . . 31 2.9.1 The Tidal Radius . . . . . . . . . . . . . . . 38

2.10 Application 2: Dark Matter Halos. . . . . . . . . . . . . 39

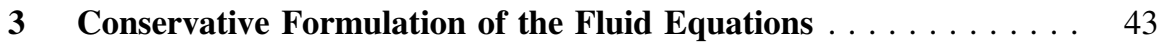

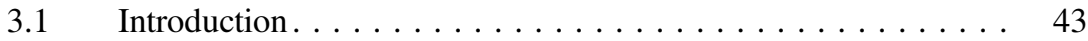

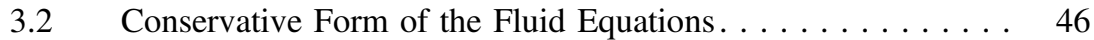

3.2.1 Conservative Mass Equation . . . . . . . . . . 47

3.2.2 Conservative Momentum Equation . . . . . . . . . . 47 
3.2.3 Conservative Form of the Energy Equation . . . . . . 48

3.2.4 Energy Non-conservation and Violent Relaxation:

A Single-Particle Analogy . . . . . . . . . . . . 52

3.3 Entropy Law for an Ideal Gas in Conservative Form . . . . . . 53

3.4 Conservative Equations with Viscosity . . . . . . . . . 54

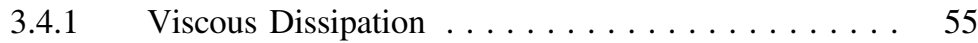

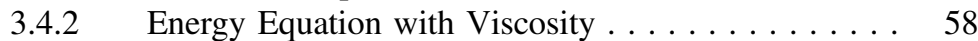

3.5 Jump Conditions and Surface Stress . . . . . . . . . . . . . . . . . . . . . 59

3.5.1 Mass Conservation . . . . . . . . . . . . . 60

3.5.2 Force Exerted by a Fluid on a Solid

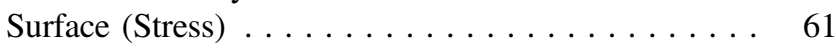

3.5.3 Conditions on a Surface Separating Two Ideal

Fluids . . . . . . . . . . . . . . . . . . . . . . . 64

4 Special Flows. . . . . . . . . . . . . . . . . . . . . . 69

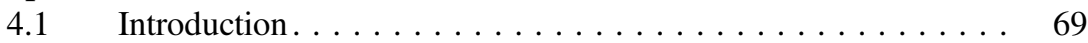

4.2 Incompressible Flows . . . . . . . . . . . . . 70

4.2.1 Stream Function for Steady Incompressible

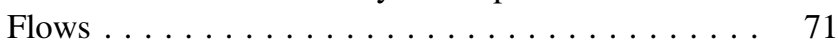

$4.3 \quad$ Irrotational Flows (Potential Flows) $\ldots \ldots \ldots \ldots . \ldots \ldots$

4.4 Bernoulli's Law for a Steady, Constant-Density Flow . . . . . . 74

5 Steady Incompressible Flows . . . . . . . . . . . . . . . . 79

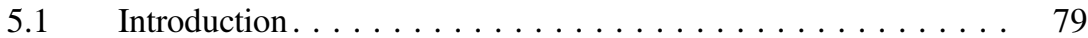

5.2 The Reynolds Number . . . . . . . . . . . . . . . . 80

5.3 Incompressible, Irrotational and Steady Corner Flow. . . . . . . 80

5.4 Laminar Viscous Flow Between Two Parallel Plates. . . . . . . 83

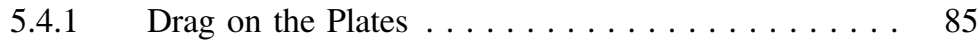

5.4.2 Case of a Moving Plate. . . . . . . . . . . . 86

5.5 Couette Flow Between Two Rotating Coaxial Cylinders . . . . 87

5.6 Small-Reynolds Number Stokes Flow Past a Sphere. . . . . . . 90

5.6.1 Drag Force on the Sphere . . . . . . . . . . . 95

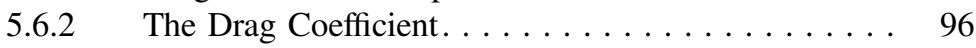

$5.7 \quad$ Ideal Potential Flow Past a Sphere . . . . . . . . . . . . . . . . . . . . . . 97

5.7.1 d'Alembert's Paradox . . . . . . . . . . . . . 99

6 Steady, Ideal Compressible Flows . . . . . . . . . . . . . . . . . . 103

$6.1 \quad$ Basic Equations . . . . . . . . . . . . . . . . 103

6.2 Bernoulli's Law for a Steady Compressible Flow . . . . . . . . . 104

6.2.1 Conservation of Entropy Along Flow Lines . . . . . . 105

6.3 The Laval Nozzle. . . . . . . . . . . . . . . . . . . 106

$6.4 \quad$ Parker's Model for a Stellar Wind . . . . . . . . . . . . . . . 108

6.4.1 Parker's Equation . . . . . . . . . . . . . . . 109

6.4.2 The Critical Point Conditions . . . . . . . . . . . 111

6.4 .3 Isothermal Winds . . . . . . . . . . . . . . 113

6.4.4 Analytical Solution for an Isothermal Wind . . . . . . 115 
6.5 The Accretion Solution . . . . . . . . . . . . . . . . 116

6.6 The Pressure-Confined Astrophysical Jet . . . . . . . . . . . . 118

6.6.1 A Bit of History. . . . . . . . . . . . . . . . . . 119

6.6.2 Fundamental Equations for a Pressure-Driven Jet

Flow . . . . . . . . . . . . . . . 120

$7 \quad$ Small Amplitude Waves: Basic Theory . . . . . . . . . . . . . . . . 125

$7.1 \quad$ Introduction. . . . . . . . . . . . . . . . . . . 125

7.1.1 Perturbation Analysis of Particle Motion

in a Potential . . . . . . . . . . . . . . . 126

7.2 What Constitutes a Small-Amplitude Wave? . . . . . . . . . . 129

7.3 The Plane Wave Representation . . . . . . . . . . . . . . . . 129

7.4 Lagrangian and Eulerian Perturbations . . . . . . . . . . 131

7.4.1 Velocity, Density and Pressure Perturbations in a Wave ........................ 132

7.5 Sound Waves....................... 143

7.6 The Plane Wave Assumption for Sound Waves . . . . . . . . . 146

7.6.1 Character of the Solutions . . . . . . . . . . . . . . 147

7.6.2 Wave Kinematics: Phase- and Group Velocity ... . . 149

7.7 Sound Waves in a Moving Fluid . . . . . . . . . . 153

$7.8 \quad$ The $\omega=0$ Solution as an Entropy Wave. . . . . . . . . . 154

8 Small Amplitude Waves: Applications. . . . . . . . . . . . . . . 157

$8.1 \quad$ The Jeans Instability . . . . . . . . . . . . . . . . . . . . . . 157

8.1.1 A Simple Physical Explanation of the Jeans

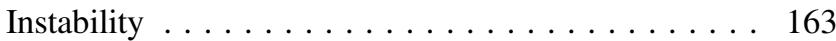

8.2 Jeans' Instability in an Expanding Universe. . . . . . . . . 165

8.2.1 Uniformly Expanding Flow . . . . . . . . . . . . 165

8.2.2 Equation for Small Perturbations . . . . . . . . . . 168

8.2.3 The Growth of the Jeans Instability. . . . . . . . . . 174

8.3 Waves in a Stratified Atmosphere. . . . . . . . . . . . . 177

8.4 Incompressible Waves. . . . . . . . . . . . . . . . . . . . 179

8.5 Compressive Waves Modified by Buoyancy .... . . . . . 182

8.5.1 The Brunt-Väisälä Frequency, Buoyancy and Convection . . . . . . . . . . . . . . . 189

8.6 Surface Waves on Water. . . . . . . . . . . . . . . . . . 192

8.6.1 Application: Kelvin Ship Waves. . . . . . . . . . 198

9 Shocks. . . . . . . . . . . . . . . . . . . . . . . . . . . 203

9.1 Introduction: What Are Shocks, and Why Do They Occur?. . . . . . . . . . . . . . 203

9.2 A Simple Mechanical Shock Analogue: The Plugged Marble-Tube . . . . . . . . . . . . . . . . . . . . . . . . . 205 
9.3 The Mathematics of Shock Formation. . . . . . . . . . . . 207

$9.3 .1 \quad$ Introduction . . . . . . . . . . . . . . . . 207

9.3.2 Characteristics and Shocks............. 208

9.3.3 Shock Formation: Getting Your Characteristics

Crossed . . . . . . . . . . . . . . . . . . 213

9.3.4 The Steepening Sound Wave once Again . . . . . . . 216

9.4 Shock Waves in a Simple Fluid . . . . . . . . . . . . . . . . . . . 219

9.5 The Weak and Strong Shock Limits . . . . . . . . . . . . . . . 224

9.6 The Rankine-Hugoniot Relations . . . . . . . . . . . . . . . . 225

9.6.1 The Limit of a Strong Shock . . . . . . . . . . . . 226

9.7 Dissipation in a Shock and the Entropy Jump . . . . . . . . . . 229

9.8 Shock Thickness and the Jump Conditions . . . . . . . . . . 230

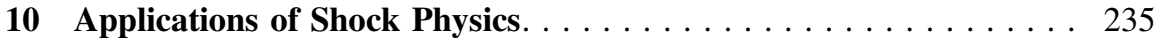

10.1 An Engineering Example: Shocks in Jet Flows . . . . . . . . 235

10.2 Astrophysical Jets. . . . . . . . . . . . . . . . . . . 236

10.3 Blowing Bubbles: Point Explosions and Winds . . . . . . . . . . 240

10.3.1 Introduction . . . . . . . . . . . . . . . . . 240

10.3.2 Expanding High-Pressure Bubbles

into a Uniform Medium. . . . . . . . . . . . . . 241

10.4 Supernova Explosions and Their Remnants . . . . . . . . . . . . . 246

10.4.1 The Core Collapse Mechanism. . . . . . . . . . . . . 246

10.4.2 Evolutionary Stages of a Supernova Remnant. . . . . 249

10.5 Stellar Wind Bubbles . . . . . . . . . . . . . . . 255

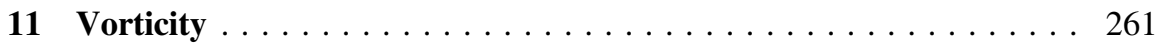

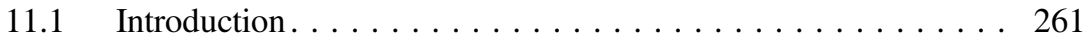

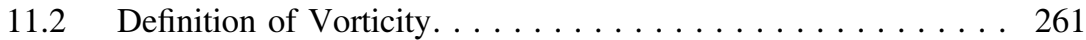

11.2.1 Vortex Stretching and Vortex Tubes . . . . . . . . . 264

11.3 Kelvin's Circulation Theorem ................. 266

11.4 Application to a Thin Vortex Tube............... 271

12 Fluid Dynamics in a Rotating Reference Frame. . . . . . . . . . . 275

12.1 Introduction. . . . . . . . . . . . . . . . . . 275

12.2 Velocity and Acceleration in a Rotating Frame . . . . . . . . . 277

12.3 Fluid Equations in a Rotating Frame. . . . . . . . . . . . 278

13 Fluids in a Rotating Frame: Applications . . . . . . . . . . . . . 281

13.1 Planetary Vorticity and the Thermal Wind Equation . . . . . . . 281

13.2 The Global Eastward Circulation in the Zonal Wind. . . . . . . . 284

13.3 The Shallow Water Approximation. . . . . . . . . . . . 286 13.3.1 The Shallow Water Equations . . . . . . . . . . . . 291

13.4 Shallow Water Waves in a Rotating Frame . . . . . . . . . . . 295 
13.5 Cyclones and Jupiter's Great Red Spot . . . . . . . . . . . . . 297

13.5.1 Cyclones . . . . . . . . . . . . . . . . . . . 297

13.5.2 Jupiter's Great Red Spot . . . . . . . . . . . . . . . 300

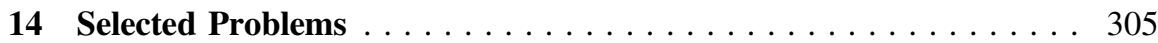

14.1 Rotation-Free, Incompressible Stagnation Flow . . . . . . . . . . . . . 305

14.2 Mass and Energy in a Corner Flow . . . . . . . . . . . . . . . . 307

14.3 The Special Status of the Comoving Derivative . . . . . . . . . 309

14.4 Flow on Cylinders . . . . . . . . . . . . . . . . 310

14.5 Use of Streamline Coordinates in Steady Flows . . . . . . . . . 312

14.6 The Isothermal Sheet . . . . . . . . . . . . . . . . . . . 315

14.7 Steady Potential Flow Past a Cylinder. . . . . . . . . . . . . . 318

14.8 Steady, Cylindrically Symmetric Constant Density Flow . . . . . 321

14.9 Radially Spreading Flow over a Plane Surface. . . . . . . . . . . 323

14.10 Applications of Bernoulli's Law. . . . . . . . . . . . . . . . 327

14.11 Steady Rotating Flow Towards a Drain. . . . . . . . . . . . . 330

14.12 Steady, Viscous Flow Along an Inclined Plane . . . . . . . . . . 331

14.13 Added Mass of an Oscillating Cylinder Immersed

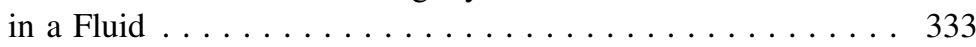

14.14 Sound Waves in a Rotating Frame . . . . . . . . . . . 336

14.15 Jeans' Instability for a Fluid Rotating on a Cylinder . . . . . . . 339

14.16 Planetary Waves. . . . . . . . . . . . . . . . . . . 341

14.17 Stellar Oscillations . . . . . . . . . . . . . . . . . . 343

14.18 Surface Waves and Surface Tension . . . . . . . . . . . . . . . . 345

14.19 The Isothermal Normal Shock . . . . . . . . . . . . . . . . . . . 348

14.20 Theory of a Hydraulic Jump in a Channel . . . . . . . . . . . . 350 14.20.1 Appendix: The Explanation for the Momentum

Conservation Law. . . . . . . . . . . . . . . . . . 354

14.21 The Kelvin-Helmholtz Instability . . . . . . . . . . . . . . . . . . . 357

14.22 Deflection is an Oblique Isothermal Shock . . . . . . . . . . . 359

14.23 The Relativistic Blast Waves of Gamma Ray Bursts . . . . . . . 361 14.23.1 Gamma Ray Bursts. . . . . . . . . . . . 361

15 Appendices .......................... 367

15.1 Mathematical Appendix: Vectors and Tensors . . . . . . . . . 367

$15.2 \quad$ Special Tensors . . . . . . . . . . . . . . . . . . . . . 369

15.3 Differential Operators . . . . . . . . . . . . . . . . 370

15.4 Directional Derivative. . . . . . . . . . . . . . . . . . 372

15.4.1 The Question of Upper and Lower Indices. . . . . . . 372

15.5 Operator Gymnastics . . . . . . . . . . . . . . . . . 373

15.6 Calculus of Differentials . . . . . . . . . . . . . . 375

15.6.1 A Word of Caution. . . . . . . . . . . . . 376 
15.7 Differential Operators in Curvilinear Coordinates . . . . . . . . 377

15.7.1 Polar Coordinates . . . . . . . . . . . . . . . 379

15.7.2 Spherical Coordinates . . . . . . . . . . . . . 380

15.8 List of Frequently Used Symbols . . . . . . . . . . . . 381

15.9 Physical and Astronomical Constants . . . . . . . . . . 383

Bibliography . . . . . . . . . . . . . . . . . . . . 385

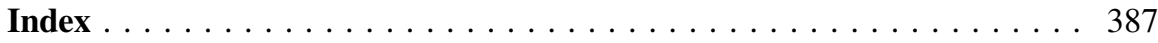

